\title{
ASSOCIAÇÃO ENTRE $O$ PADRÃO DE ATIVIDADE FÍSICA MATERNA, GANHO PONDERAL GESTACIONAL E PESO AO NASCER EM UMA COORTE DE 118 GESTANTES NO MUNICÍPIO DE CAMPINA GRANDE, NORDESTE DO BRASIL
}

\author{
Jousilene de Sales Tavares ${ }^{1}$, Adriana Suely de Oliveira Melo², Melania Maria Ramos de Amorim*3, Viviane de Oliveira Barros ${ }^{4}$, Maria Helena \\ D'Aquino Benício ${ }^{5}$, Monica Yuri Takito ${ }^{5}$, Maria Aparecida Alves Cardoso ${ }^{6}$ \\ Trabalho desenvolvido no Núcleo de Estudos e Pesquisas Epidemiológicas (NEPE) da Universidade Estadual da Paraíba Instituto Materno Infantil Professor \\ Fernando Figueira (IMIP)
}

*Correspondência:

Rua Neusa Borborema de Souza, 300

Bairro Santo Antônio

Campina Grande - PB

CEP 58103-313

\begin{abstract}
RESUMO
OвJetivo. Esta pesquisa estudou o efeito da atividade física sobre o ganho ponderal e peso ao nascer em gestantes do Programa Saúde da Família no município de Campina Grande, (PB).

MÉtodos. Realizou-se um estudo de coorte abrangendo 118 gestantes, entre 2005 e 2006. A avaliação do estado nutricional inicial seguiu os critérios de Atalah e o ganho ponderal materno foi avaliado de acordo com o Institute of Medicine. Analisou-se o padrão de atividade física de acordo com o medidor de estimativa de gasto calórico total (METs) e sua associação com o ganho ponderal e o peso ao nascer. Resultados. 0 padrão de atividade física predominante na $16^{a}$ semana foi o leve, alcançando 85,6\% das gestantes. Observou-se uma redução importante do nível de atividade física com a evolução da gestação, com $98,3 \%$ das gestantes sendo consideradas sedentárias na $24^{\text {a }}$ semana e $100 \%$ na $32^{\text {a }}$ semana. No momento da captação, cerca de $50 \%$ das gestantes eram eutróficas, $23 \%$ com baixo peso e as demais com sobrepeso/obesidade. No segundo e no terceiro trimestres, aproximadamente $45 \%$ das gestantes ganharam peso excessivo. A distribuição do peso ao nascer mostrou um predomínio do peso adequado (85,6\%), e uma alta prevalência de macrossomia (8,5\%). Dentre os desfechos avaliados, foi observada associação apenas entre os valores de METs da $24^{\text {a }}$.semana e o ganho ponderal materno no segundo trimestre gestacional $(p=0,045)$.

ConcLusÃo. Todas as gestantes estavam sedentárias no terceiro trimestre; observou-se uma associação entre o padrão de atividade física e o ganho ponderal materno no segundo trimestre, mas não houve associação deste padrão com o peso ao nascer.
\end{abstract}

UnIteRmos: Atividade motora. Alterações do ganho ponderal. Gravidez. Peso ao nascer.

\section{INTRODUÇÃO}

A atividade física é definida como qualquer movimento corporal, produzido pelos músculos esqueléticos, que resulte em gasto energético maior que os níveis de repouso ${ }^{1}$. Conceitualmente, diferencia-se do exercício físico que se caracteriza como uma atividade física planejada, estruturada e realizada de forma sistemática com o objetivo de melhorar ou manter a aptidão física. Portanto, embora todo exercício físico seja uma atividade física, nem toda atividade física é um exercício.

Ressaltando a importância da prática de atividade física regular em todo o ciclo vital, instituições e órgãos responsáveis pela saúde pública vêm buscando estabelecer diretrizes para a promoção de estilos de vida saudáveis, procurando definir critérios para a sistematização da atividade física quanto à duração, intensidade e frequência ${ }^{2}$.

Além de atuar na prevenção e no controle de diversas doenças, a prática da atividade física melhora a qualidade de vida, inclusive para grupos especiais, dentro dos quais se incluem as gestantes ${ }^{3}$. Até pouco tempo, as recomendações limitavam a prática de atividade física para este grupo ou até mesmo as contraindicava ${ }^{4,5}$. Hoje, já existe consenso sobre a importância da atividade física durante a gestação, embora alguns efeitos ainda estejam em estudo. Dentre estes, se destacam o controle do ganho ponderal gestacional materno e a sua influência sobre o peso ao nascer.

1. Mestre em Saúde Coletiva - Pesquisadora do NEPE - UEPB. Campina Grande, PB

2. Mestre em Saúde Coletiva - Aluna do Doutorado em Saúde Materno-Infantil do IMIP. Recife, PE

3. Doutora em Medicina subárea Tocoginecologia pela UNICAMP - professora da pós-graduação do Instituto Materno Infantil Professor Fernando Figueira, Recife, PE

4. Mestre em Saúde Coletiva - Pesquisadora do NEPE, Campina Grande, PB

5. Doutora em Saúde Pública - Professora da USP, São Paulo, SP

6. Doutora em Saúde Pública - Professora da UEPB, Campina Grande, PB 
Em relação aos efeitos da prática da atividade física sobre o ganho ponderal na gestação observam-se duas vertentes, demonstrando que esta causalidade ainda precisa ser melhor estudada. Há estudos demonstrando o seu efeito sobre o ganho de peso, enquanto que outros não confirmam tal associação. Um estudo longitudinal verificou que a prática de atividade física recreacional reduziu a velocidade do ganho de peso gestacional e diminuiu a gordura localizada ${ }^{6,7}$. Confirmando esses achados, outro estudo encontrou que a atividade física através da prática intencional da caminhada reduz a frequência de obesidade entre as gestantes ${ }^{6,7}$. Entretanto, existe um estudo em que só se observou redução do ganho ponderal entre as gestantes previamente ativas, que inicialmente reduziram a frequência da atividade física, aumentando-a em seguida no decorrer da gravidez ${ }^{3}$, enquanto outro não demonstrou nenhuma associação entre atividade física e ganho ponderal ${ }^{8}$.

Preocupação maior refere-se ao crescimento fetal e peso ao nascer, uma vez que o efeito da atividade física sobre esses desfechos tem gerado controvérsias. Se por um lado alguns estudos sugerem que a atividade física promove um maior ganho de peso fetal, principalmente quando iniciada precocemente ${ }^{9,10}$, por outro lado há estudos demonstrando uma redução no peso dos recém-nascidos cujas mães eram praticantes de atividade física durante a gestação $0^{11,12}$.

Várias técnicas são utilizadas com a finalidade de avaliar o padrão de atividade física de um indivíduo, sendo relatadas na literatura mais de 30 técnicas diferentes. Os instrumentos de medida podem ser classificados, de modo geral, em: os que utilizam as informações oriundas dos sujeitos (questionários, entrevistas e diários, a partir dos quais pode ser calculado o gasto energético) e os marcadores fisiológicos e sensores de movimento ${ }^{13}$

Buscando contribuir com esse debate e considerando a carência de estudos realizados no Brasil, e em particular a ausência de pesquisas sobre o tema no Nordeste até o momento, este estudo visa a avaliar o efeito da atividade física sobre o ganho ponderal materno e sobre o peso dos recém-nascidos de gestantes atendidas pelo Programa Saúde da Família (PSF) no município de Campina Grande (PB).

\section{MÉtodos}

Realizou-se um estudo de coorte prospectivo, desenvolvido dentro do projeto "Impacto da orientação alimentar e da atividade física sobre o fluxo sanguíneo feto-placentário e crescimento fetal" O projeto maior foi constituído por duas coortes, a primeira observacional e a segunda de intervenção (orientação para a prática de atividade física sem supervisão), sendo utilizados para este artigo os dados da primeira coorte. A coorte observacional incluiu 118 gestantes, captadas nas unidades do PSF de Campina Grande, entre março de 2005 e maio de 2006. O projeto de pesquisa foi aprovado pelo Comitê de Ética da Universidade Estadual da Paraíba e seguiu as diretrizes éticas de pesquisa com seres humanos, resolução 196/96 do Conselho Nacional de Saúde.

Um dos objetivos do projeto maior foi avaliar o impacto da atividade física sobre o ganho ponderal gestacional, e, portanto, a amostra foi calculada com base na prevalência de ganho de peso excessivo observada em estudos realizados nos últimos anos no Brasil, permitindo detectar prevalências de até 30\%, com precisão absoluta de oito pontos percentuais e intervalo de confiança igual a 95\%, podendo detectar coeficientes de correlação de "0,25 ou maior", com poder de $80 \%$ e erro alfa de 0,05.

Adotaram-se como critérios de inclusão a idade materna igual ou superior a 18 anos, a idade gestacional máxima de 16 semanas e gestação única. Foram excluídas gestantes com as seguintes condições: doenças maternas crônicas, anomalias ou perdas fetais.

A cada quatro semanas as gestantes respondiam a questionários pré-testados abordando informações obstétricas, socioeconômicas e clínicas. O padrão de atividade física cotidiano foi avaliado através de um questionário específico para gestantes brasileiras, desenvolvido e validado por Takito et al. ${ }^{14}$ e aplicado em três momentos do seguimento, na $16^{a}, 24^{a}$ e $32^{a}$ semanas gestacionais, com o objetivo de avaliar o padrão de atividade física em períodos diferentes.

As variáveis antropométricas utilizadas foram o peso e a estatura, adotando-se as técnicas padronizadas recomendadas por Jellife e Jellife ${ }^{15}$. O peso foi aferido por balança antropométrica portátil digital, com acuidade de 200 gramas (Tanita) e, para a estatura foi utilizado um antropômetro móvel (Seca) com acuidade de 1 centímetro.

A partir da altura e do peso mensurados no primeiro encontro foi calculado o índice de massa corporal (IMC) (peso/altura²), o qual foi utilizado para classificar as gestantes de acordo com o estado nutricional inicial em: baixo peso, eutrofia, sobrepeso e obesidade. Para esta classificação foram considerados os níveis críticos do IMC para a idade gestacional, propostos por Atalah et al $^{16}$, adotados pelo Ministério da Saúde.

O ganho ponderal no segundo e terceiro trimestres foram obtidos através da diferença entre os pesos das gestantes nas $28^{a}$ e $16^{a}$ semanas e nas $28^{a}$ e $36^{a}$ semanas gestacional, respectivamente. Para classificar a adequação do ganho de peso gestacionais, no segundo e no terceiro trimestres, foram utilizados os critérios do Institute of Medicine ${ }^{17}$ de acordo com o estado nutricional inicial da gestante. Ao final do seguimento, as gestantes foram classificadas nas seguintes categorias: ganho de peso insuficiente, adequado ou excessivo.

A atividade física foi medida através do cálculo do "METs" (medidor de estimativa de gasto calórico total). Uma variável denominada de METgest foi criada para estimar o padrão de atividade física das gestantes. Inicialmente estimou-se a frequência semanal e a duração (em minutos) das atividades cotidianas, laborais e caminhadas cotidianas. A seguir, multiplicou-se a frequência de cada atividade pela sua duração em minutos, obtendo-se um total de tempo em uma semana (TTS) gasto pela gestante com essa atividade. Para elaborar o estimador, aqui denominado METgest, o TTS de cada atividade foi multiplicado pelo MET referente a essa atividade de acordo com o compendium de atividade física ${ }^{18}$, obtendo-se um TTS ponderado pelo MET para cada atividade (TTSP). O METgest, então, foi calculado dividindo-se o somatório ponderado de TTSs $\left(\Sigma_{\text {TTSP }}\right)$ pelo somatório de tempo das atividades em uma semana $\left(\Sigma_{\mathrm{TTS}}\right)$. Para este cálculo foi utilizada a seguinte fórmula:

\section{METgest $=\Sigma_{\mathrm{TTSP}} / \Sigma_{\mathrm{TTS}}$}

Onde: $\mathrm{TTSP}_{\mathrm{i}}=\mathrm{TTS}_{\mathrm{i}} \times \mathrm{MET}_{\mathrm{i}}$

$\mathrm{TTS}_{\mathrm{i}}=\mathrm{F}_{\mathrm{i}} \times \mathrm{T}_{\mathrm{i}}$ 
$\mathrm{F}=$ frequência de cada atividade

$\mathrm{T}=$ tempo gasto com cada atividade

= índice i para cada atividade cotidiana

TTSP = frequência de cada atividade pela sua duração em

minutos multiplicada pelo índice da atividade

TTS = frequência de cada atividade pela sua duração em minutos

A classificação das gestantes de acordo com o padrão da atividade física baseou-se nos níveis de intensidade de METgest, método adaptado de Chasan-Taber et a ${ }^{19}$. Os valores de METGest menores que 1,5 classificam a gestante como sedentária; entre 1,5 a 2,9 equivale ao padrão de atividade física leve; entre 3 e 5,9 o padrão é considerado moderado e maior ou igual a 6 vigoroso.

Coletaram-se dados referentes aos recém-nascidos (RNs) nos prontuários das maternidades. O peso ao nascer foi classificado segundo os critérios da Organização Mundial da Saúde (OMS) em: baixo peso (RN com menos de $2.500 \mathrm{~g}$ ), peso adequado (RN com peso entre 2.500 e $3.999 \mathrm{~g}$ ), e excesso de peso ou macrossomia (RN com $4.000 \mathrm{~g}$ ou mais). A escolha do peso absoluto em detrimento das categorias de peso ao nascer (baixo peso, peso adequado e macrossomia) deveu-se ao fato de a amostra estudada ser de baixo risco, esperando-se, portanto uma baixa prevalência de RNs pequenos para a idade gestacional, inviabilizando uma busca de associação.

A análise estatística foi realizada nos programas Epi Info versão 3.4.1 e STATA versão 7.0, considerando-se o nível de significância estatística de 5\%. Após a análise descritiva das principais variáveis do estudo foram realizados testes de hipóteses para avaliar a existência de associação entre a variável contínua METs em relação aos desfechos peso ao nascer e ganho ponderal gestacional para as $16^{\mathrm{a}}, 24^{\mathrm{a}}$ e $32^{\mathrm{a}}$ semanas gestacionais. Utilizaram-se os testes não paramétricos de Kruskal Wallis (variáveis com três categorias) e o de Mann-Whitney (duas categorias) para a análise da atividade física referente à $24^{\text {a }}$ semana, uma vez que esta não mostrou distribuição normal. Para a $16^{\mathrm{a}}$ e a $32^{\mathrm{a}}$ semanas utilizou-se análise de variância.

0 efeito da atividade física sobre o peso ao nascer foi avaliado inicialmente para as categorias de peso inadequado, ou seja, para o baixo peso ao nascer e macrossomia e, em seguida, através de análise de regressão linear para a variável peso ao nascer enquanto variável desfecho contínua. 0 modelo de regressão foi ajustado por renda familiar per capita, idade materna, idade gestacional ao nascimento e ganho de peso em categorias para o segundo trimestre gestacional.

$O$ efeito dos METs sobre o ganho ponderal materno foi testado em relação às categorias de ganho de peso insuficiente, adequado e excessivo para o segundo e terceiro trimestres, respectivamente. Para se avaliar de forma mais específica, nas situações em que as categorias mostraram associação estatística $(p<0,05)$, elaboraram-se modelos de regressão multinomial para as três categorias de ganho de peso: insuficiente e excessivo tendo-se como referência o grupo de ganho de peso normal. Esses modelos foram ajustados pela renda per capita e estado nutricional inicial da gestante.

\section{Resultados}

A coorte incluiu 137 gestantes, tendo ocorrido $13,9 \%$ de perdas de seguimento resultando em uma amostra de 118
Figura 1 - Organograma das exclusões e perdas da coorte

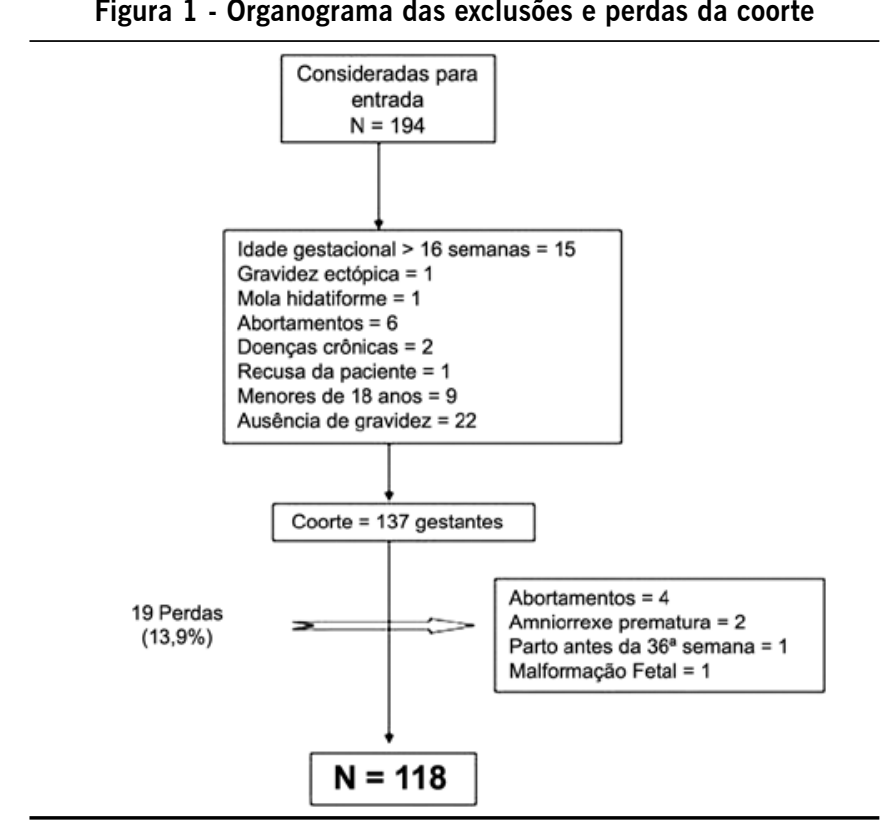

(Figura 1). Não foram observadas diferenças entre as gestantes incluídas no estudo e as perdas em relação às principais características socioeconômicas e ao estado nutricional materno inicial.

As características da coorte em relação às variáveis socioeconômicas e demográficas indicam que $24,3 \%$ das gestantes estavam dentro da faixa etária de 18 a 20 anos, e a idade média foi de 23 anos. Em relação à escolaridade, mais da metade das participantes não atingiram o segundo grau. Cerca de $92 \%$ das gestantes possuíam renda per capita abaixo de um salário mínimo e $64,3 \%$ não trabalhavam fora de casa.

A distribuição das gestantes de acordo com as variáveis reprodutivas indica que $66,1 \%$ eram multigestas. Em relação ao intervalo interpartal, 40,8\% das mulheres apresentaram um intervalo maior ou igual a dois e menor ou igual a cinco anos, e aproximadamente $90 \%$ das gestantes realizaram mais de seis consultas médicas durante o pré-natal. A prevalência de tabagismo foi de aproximadamente $8 \%$.

De acordo com as categorias do estado nutricional, no momento da captação, $50 \%$ das gestantes eram eutróficas, $23 \%$ estavam com baixo peso e as demais com sobrepeso (19\%) e obesidade (8\%). A evolução do ganho ponderal no segundo e terceiro trimestres evidenciou uma frequência de $13 \%$ e $23 \%$ de gestantes com ganho ponderal insuficiente, $44 \%$ e $32 \%$ de gestantes com ganho ponderal adequado e $43 \%$ e $45 \%$ com ganho de peso excessivo, respectivamente.

A distribuição das gestantes de acordo com o padrão de atividade física para as semanas correspondentes aos trimestres gestacionais $\left(16^{\mathrm{a}}, 24^{\mathrm{a}}\right.$ e $32^{\mathrm{a}}$ semanas) indica uma evidente redução da atividade ao longo do período gestacional $(p<0,05)$; observa-se que já no segundo trimestre as gestantes se tornam sedentárias.

A distribuição do peso ao nascer da coorte de nascimentos, segundo os critérios da OMS, mostrou uma prevalência de $85,6 \%$ de peso adequado, $8,5 \%$ de macrossomia e $5,9 \%$ de 
TAVARES JS ET AL.

baixo peso. Foram observados três casos de parto prematuro $(2,5 \%)$, não sendo verificada associação com o padrão de atividade física (dados não apresentados em tabela). Não se observou associação estatisticamente significante entre as médias dos METs das atividades físicas realizadas pelas mães nos trimestres gestacionais e as categorias de baixo peso ao nascer e macrossomia (Tabela 1). A análise de regressão linear multivariada confirmou a inexistência desta associação (dados não apresentados em tabela).

A análise do efeito da atividade física sobre o ganho de peso materno indicou a existência de associação apenas entre os valores de METs da $24^{\mathrm{a}}$ semana sobre o ganho ponderal materno no segundo trimestre gestacional $(p=0,045)$ (Tabela 1$)$. Esta associação permaneceu no modelo de regressão multinomial $(p=0,012)$ (dados não apresentados em tabelas).

\section{Discussão}

Os resultados evidenciam que a coorte estudada apresentava as características peculiares às populações de gestantes atendidas por serviços públicos de saúde. Uma vez que a amostra foi composta em sua maioria de gestantes residentes em áreas mais carentes da cidade, confirmaram-se as prevalências esperadas de baixo poder aquisitivo, baixa escolaridade e baixo percentual de mulheres economicamente ativas.

Em relação ao padrão de atividade física, percebe-se a alta prevalência do padrão sedentário e baixo percentual do padrão moderado e a ausência do padrão vigoroso de atividade física em todas as idades gestacionais estudadas na coorte. Estes achados demonstram um estilo de vida inadequado por parte das gestantes estudadas, com alta prevalência do sedentarismo, proporcionando uma redução no gasto energético. Esta redução, associada ao aumento do consumo de calorias vazias, evidenciado por estudos nesta população ${ }^{20,21}$, podem favorecer um desequilíbrio do consumo e do gasto, resultando em maior ganho ponderal, com repercussões para a mãe e o feto ${ }^{5}$.

Analisando-se o ganho ponderal das gestantes, observou-se uma alta prevalência de ganho de peso excessivo no segundo e no terceiro trimestres, mesmo tendo sido utilizados os critérios do IOM. Estes critérios de adequação do ganho ponderal têm sido amplamente criticados, pois tendem a considerar como ganho adequado um número maior de gestantes, uma vez que um ganho de até $16 \mathrm{~kg}$ é considerado adequado. Esta alta prevalência de ganho de peso gestacional acima do recomendado tem sido observada em vários estudos realizados tanto em países desenvolvidos como em desenvolvimento ${ }^{22-24}$. Outro fator preocupante foi o alto percentual de gestantes com sobrepeso/ obesidade já no início da gestação, superando o percentual de desnutridas, mesmo em uma região pobre como a Nordeste. Estes dados sugerem que mesmo em regiões mais pobres a transição nutricional já está evidente, o que deve motivar os órgãos envolvidos com a saúde pública a estimular um estilo de vida mais saudável na tentativa de controlar o ganho excessivo de peso. Dentro dos hábitos saudáveis, destacam-se a orientação alimentar, evitando-se o excesso no consumo de calorias vazias e a prática de atividade física. Entretanto ainda não está claro o real impacto da atividade física sobre este desfecho.

Neste estudo, observou-se associação apenas entre a atividade física realizada pelas gestantes na $24^{a}$ semana com o ganho
Figura 2 - Distribuição das gestantes da coorte de acordo com 0 padrão de atividade física por trimestre gestacional

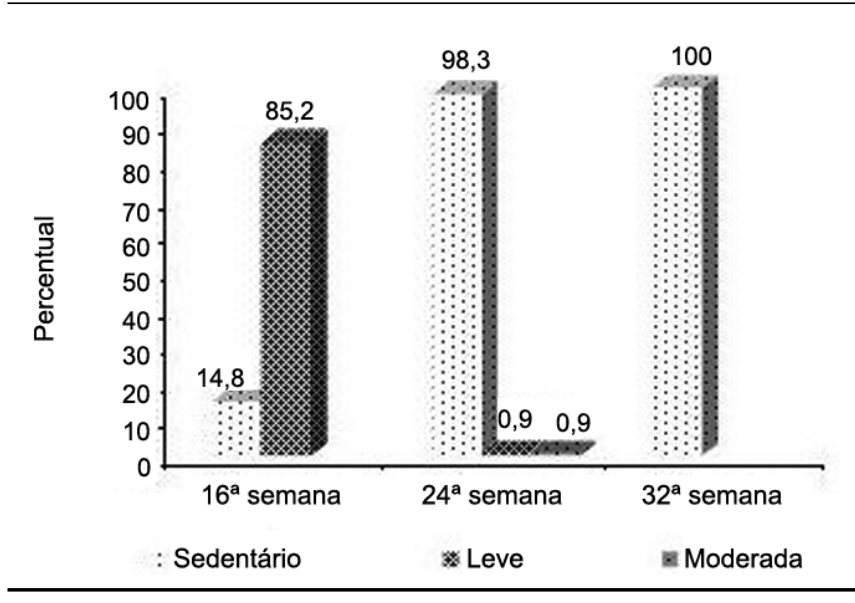

ponderal no segundo trimestre. Apesar da associação observada, alguns questionamentos devem ser levantados, uma vez que o resultado encontrado foi o oposto ao observado em outros estudos. Esperava-se que, se fosse observado algum efeito da atividade física sobre o ganho ponderal, seria que quanto maior o padrão de atividade física maior seria o risco de ganho de peso insuficiente. Entretanto, no presente estudo, as gestantes com ganho de peso insuficiente tiveram uma menor média de METs, ou seja, uma menor intensidade de atividade física. Este fato pode ser justificado pela grande quantidade de gestantes sedentárias observadas no estudo, favorecendo o aparecimento de associações espúrias, sem significado clínico. Outro fato que pode justificar tal associação foi o uso de variáveis contínuas, que pode ter favorecido obter-se uma significância estatística na diferença de médias, sem relevância clínica, uma vez que observamos que a variação da média dos METs, neste caso, não extrapola o ponto de corte do sedentarismo.

A associação entre atividade física e ganho ponderal na gestação não foi observada em estudo de coorte envolvendo mulheres sedentárias e ativas ${ }^{8}$. Em contrapartida, em um ensaio clínico avaliando gestantes previamente ativas que iniciaram um programa de exercício sistemático com redução da frequência no início do programa, seguida por aumento no decorrer da gravidez, os autores observaram uma redução no ganho ponderal durante a gestação ${ }^{9}$. Um estudo de intervenção realizado no Brasil, avaliando o efeito da hidroterapia sobre o ganho ponderal e a quantidade de gordura materna, não constatou diferenças entre o ganho de peso gestacional nos dois grupos (hidroterapia e controle $)^{25}$. Entretanto, os autores verificaram que os índices de massa magra aumentaram de modo significativo entre o início e o final da gestação e, apesar do aumento significativo de gordura absoluta, a proporção peso/gordura foi mantida. Estes achados reforçam a ideia de que a atividade física tem que ser sistematizada e monitorizada para melhor esclarecer o seu efeito, o que não foi realizado neste estudo.

Em relação ao peso ao nascer, observou-se um predomínio do peso adequado, entretanto merece destaque o alto percentual de macrossomia observado, bem acima da média nacional que é de 
Tabela 1 - Associação entre a atividade física em METs na $16^{\mathrm{a}}, 24^{\mathrm{a}}$ e $32^{\mathrm{a}}$ semanas com o baixo peso ao nascer, a macrossomia e o ganho ponderal no segundo e terceiro trimestres gestacionais

\begin{tabular}{|c|c|c|c|}
\hline & $16^{\mathrm{a}}$ semana & $24^{\mathrm{a}}$ semana & $32^{\mathrm{a}}$ semana \\
\hline Variáveis & $\begin{array}{c}\text { METs } \\
\text { Média } \pm \text { DP }\end{array}$ & $\begin{array}{c}\text { METs } \\
\text { Média } \pm \text { DP }\end{array}$ & $\begin{array}{c}\text { METs } \\
\text { Média } \pm \text { DP }\end{array}$ \\
\hline \multicolumn{4}{|c|}{ Peso ao Nascer } \\
\hline Sim & $1,79 \pm 0,43$ & $0,71 \pm 0,27$ & $0,59 \pm 0,12$ \\
\hline Não & $1,90 \pm 0,38$ & $0,68 \pm 0,36$ & $0,58 \pm 0,14$ \\
\hline$p$ & $0,67^{*}$ & $0,48 * *$ & $0,93 *$ \\
\hline \multicolumn{4}{|c|}{ Baixo peso ao nascer } \\
\hline Sim & $1,85 \pm 0,34$ & $0,56 \pm 0,12$ & $0,55 \pm 0,16$ \\
\hline Não & $1,90 \pm 0,38$ & $0,69 \pm 0,36$ & $0,58 \pm 0,13$ \\
\hline$p$ & $0,80 *$ & $0,26 * *$ & $0,43^{*}$ \\
\hline \multicolumn{4}{|c|}{ Ganho ponderal } \\
\hline Insuficiente & $1,97 \pm 0,40$ & $0,53 \pm 0,10$ & $0,56 \pm 0,14$ \\
\hline Adequado & $1,92 \pm 0,40$ & $0,69 \pm 0,37$ & $0,58 \pm 0,14$ \\
\hline Excessivo & $1,85 \pm 0,35$ & $0,71 \pm 0,36$ & $0,58 \pm 0,13$ \\
\hline$p$ & 0,40 & $0,045^{* * *}$ & 0,97 \\
\hline \multicolumn{4}{|c|}{ No terceiro trimestre } \\
\hline Insuficiente & $1,99 \pm 0,45$ & $0,63 \pm 0,18$ & $0,56 \pm 0,16$ \\
\hline Adequado & $1,86 \pm 0,32$ & $0,71 \pm 0,43$ & $0,58 \pm 0,13$ \\
\hline Excessivo & $1,87 \pm 0,38$ & $0,68 \pm 0,35$ & $0,59 \pm 0,13$ \\
\hline$p$ & 0,42 & $0,98^{* * *}$ & 0,49 \\
\hline
\end{tabular}

* Teste T de student

** Teste de Mann-Whitney

*** Teste não paramétrico Kruskal Wallis

$5 \%{ }^{26,27}$. Este resultado pode ser justificado pelo alto percentual de ganho ponderal excessivo pelas gestantes. Este aumento na prevalência da macrossomia também vem sendo observado em outros estudos ${ }^{28,29}$ e alerta a comunidade científica sobre o crescente aumento da obesidade, com o processo iniciando ainda na vida intrauterina. Além dos efeitos sobre o ganho de peso gestacional, também se questionam os efeitos da atividade física em relação ao peso ao nascer. Sabe-se atualmente que um dos fatores principais associado ao peso ao nascer é o ganho ponderal materno. Desta forma, só se esperava encontrar interferência no peso ao nascer se tivéssemos observado efeito do padrão de atividade física sobre o ganho ponderal.

No presente estudo não se encontrou associação estatisticamente significante entre a atividade física e o peso ao nascer. A ausência de associação também foi observada em um estudo de coorte que acompanhou dois grupos de gestantes (praticantes vigorosas e não vigorosas de atividade física), realizado com o objetivo de avaliar o impacto da atividade física sobre o peso ao nascer ${ }^{30}$. Por outro lado, em uma coorte de 800 gestantes distribuídas em três grupos: praticantes com intensidade leve/ moderada, praticantes com intensidade alta e não praticantes, verificou-se que as gestantes que se exercitavam durante os três trimestres tenderam a ter bebês com peso maior ${ }^{31}$. Resultado semelhante foi observado em um ensaio clínico envolvendo 46 gestantes previamente sedentárias, cujo objetivo avaliar era 0 impacto da atividade física sobre o peso ao nascer ${ }^{9}$. Os autores observaram que gestantes que realizavam exercício com maior frequência no início da gestação tinham maior probabilidade de ter filhos com maior peso ao nascer.

Um estudo de coorte realizado no Brasil, com o intuito de verificar o efeito da atividade física sobre o peso ao nascer, não observou associação entre estas variáveis. No entanto, foi detectada associação com a postura parada em pé, ou seja, recémnascidos de gestantes que permaneciam paradas em pé mais de duas horas e meia por dia no segundo trimestre, apresentaram um maior risco de peso ao nascer inadequado (OR ajustado =3,23; IC 95\%: 1,30-7,99) ${ }^{14}$.

Uma das limitações deste estudo foi o fato de a avaliação do padrão da atividade física ter sido obtida através de questionários e não monitorizada através de sensores de movimentos. Entretanto, a avaliação do padrão de atividade física através dos questionários é amplamente utilizada em estudos nacionais e internacionais, existindo inclusive artigo comparando a aplicação de questionário e utilização de sensores de movimentos para a avaliação do padrão de atividade física, sem diferenças estatisticamente significantes ${ }^{32}$. Com base nestes artigos, desenhamos este estudo, porém os resultados não foram significativos, apesar dos cuidados na avaliação. Conclui-se, portanto, que trabalhos com objetivo de avaliar o impacto da atividade sobre desfechos gestacionais e perinatais, devem extrapolar a simples avaliação, estimulando uma prática de exercício contínua, monitorizada e sistematizada. 


\section{Conclusões}

Conclui-se que a falta de associação entre a atividade física e o peso ao nascer pode ter se devido à elevada frequência de sedentarismo entre as gestantes. Estudos mostram que os efeitos da atividade física sobre os desfechos peso ao nascer e ganho ponderal materno só são alcançados quando a atividade física é executada de forma moderada, categoria praticamente não observada nesta população ${ }^{30,11}$.

Estudos posteriores e mais específicos são necessários para avaliar o efeito da atividade física na gravidez sobre o ganho de peso gestacional e o crescimento/peso fetal, controlandose para que esta seja realizada de forma regular, moderada e sistematizada. Este tipo de pesquisa idealmente deve ser conduzido através de ensaios clínicos randomizados, permitindo dimensionar de maneira mais adequada os fatores envolvidos na causalidade, além de uma melhor avaliação dos desfechos, na medida em que se diminuem os efeitos confundidores envolvidos no processo, que é bastante complexo. Um ensaio clínico com este objetivo encontra-se atualmente em andamento na cidade de Campina Grande, prevendo-se a inclusão de 150 gestantes.

Conclusão mais importante ainda, oriunda deste trabalho, é que o nível de sedentarismo entre as gestantes foi alarmante, sugerindo que novas pesquisas sejam realizadas com a finalidade de detectar se este é um fenômeno global ou isolado, e a partir daí desenvolver políticas públicas voltadas para esta realidade.

\section{SUPORTE FinAnCEIRO:}

$\mathrm{CNPq}$

\section{Conflito de interesse: não há}

\section{SUMMARY}

Association betWeen maternal physical activity, gestational WEIGHT GAIN AND BIRTH WEIGHT IN A COHORT OF 118 PREGNANT WOMEN in Campina Grande, Northeast of Brazil

OBJECTIVE. This survey evaluated the effects of physical activity on gestational weight gain and birth weight of pregnant women attended by the Family Health Program in Campina Grande, Northeast of Brazil.

Methods. A cohort study enrolling 118 pregnant women was conducted between 2005 and 2006. Evaluation of the initial nutritional status was performed following Atalah's criteria and gestational weight gain was evaluated according to the Institute of Medicine. The pattern of physical activity was evaluated according to METs (metabolic equivalent) and its association with weight gain and birth weight was determined.

RESULTS. The predominant physical activity pattern at 16 weeks was mild, in $85.6 \%$ of pregnant women. An important reduction of physical activity levels was observed with evolution of pregnancy and $98.3 \%$ of pregnant women were sedentary at 24 weeks and $100 \%$ at 32 weeks. In the initial evaluation of nutritional status 50\% were normal, 23\% had low weight and $27 \%$ had overweight/obesity. At second and third trimesters about $45 \%$ of pregnant women had excessive weight gain. Adequate birth weight for gestational age was found in $85.6 \%$ of neonates but macrosomia was highly frequent (8.5\%). A signi- ficant association between METs at 24 weeks and gestational weight gain was found in the second trimester $(p=0.045)$.

CONCLUSION. All the pregnant women were sedentary in the third trimester and a significant association between physical activity pattern and gestational weight gain was found in the second trimester, but there was no association between this pattern and birth weight. [Rev Assoc Med Bras 2009; 55(3): 335-41]

KEY WORDS: Motor activity. Body weight changes. Pregnancy. Birth weight.

\section{REFERÊNCIAS}

1. Caspersen CJ, Kriska AM, Dearwater SR. Physical activity epidemiology as applied to elderly populations. Baillieres Clin Rheumatol. 1994;8:7-27.

2. World Health Organization (WHO). The optimal duration of exclusive breastfeeding a systematic review. Geneva: World Health Organization; 2002.

3. Clapp JF, Kim H, Burciu B, Lopez B. Beginning regular exercise in early pregnancy: effect on fetoplacental growth. Am J Obstet Gynecol. 2000; 183:1484-8.

4. ACOG. American College of Obstetricians and Gynecologists. Exercise during pregnancy and the postpartum period. Washington (DC): ACOG Technical Bulletin; 1994. p.189.

5. ACOG. American Colloge of Obstetricians and Gynecologists. Committee on Obstetric. Exercise during pregnancy and the postpartum period. Pratice n. ${ }^{\circ}$ 267. Am Col Obstet Gynecol. 2002;99:171-3.

6. Clapp, JF; Litlle, KD. Effect of recreational exercise on pregnancy weight gain and subcutaneous fat deposition. Med Sci Sports Exerc. 1995;27:170-7.

7. Matsudo VKR, Matsudo SMM. Atividade física e esportiva na gravidez. In: Tedesco JJ, editor. A grávida. São Paulo: Atheneu; 2000. p.53-81.

8. Horns PN, Ratcliffe LP, Leggett JC. Pregnancy outcomes among active and sedentary primiparous women. J Obstet Gynecol Neonatal Nurs. 1996;25:49-54.

9. Clapp JF, Kim H, Burciu B. Continuing regular exercise during pregnancy: effect of exercise volume on fetoplacental growth. Am J Obstet Gynecol. 2002;186:142-7.

10. Clapp JF. The effects of maternal exercice on fetal oxygenation and fetoplacental growth. J Obstet Gynecol Reprod Biol. 2003; 110(Suppl 1):s80-5.

11. Klebanoff MA, Shiono PH, Carey JC. The effect of physical activity during pregnancy on preterm delivey and bith weight. Am J Obstet Gynecol. 1990;163:1450-6.

12. Campbell MK, Mottola MF. Recreational exercise and occupational activity during pregnancy and birth weight: a case-control study. Am J Obstet Gynecol. $2001 ; 184: 403-8$.

13. Reis RS, Petroski EL, Lopes AS. Medidas da atividade física: revisão de métodos. Rev Bras Cineantropometr Desemp Hum. 2000;2:89-96.

14. Takito MY, Benício MHDA, Latorre MRDO. Postura materna durante a gestação e sua influência sobre o peso ao nascer. Rev Saude Pública. 2005;39:325-32.

15. Jellife DB, Jellife EFP. Community nutritional assessment. New York: Oxford University Press; 1989.

16. Atalah E, Castillo C, Castro R, Aldeã A. Propuesta de um nuevo estándar de evoluación nutricional em embarazadas. Rev Med Chile. 1997;125:1429-36.

17. IOM. Institute of Medicine. Subcommittee on nutritional status and weight gain during pregnancy. Nutrition during pregnancy. Washington (DC): National Academy Press; 1990.

18. Ainsworth BE, Haskell, WL, Whitt MC, Irwin ML, Swartz AM, Strath SJ, et al. Compendium of physical activities: an update of activity codes and met intensites. Med Sci Sports Exerc. 2000;32:498-516.

19. Chasan-taber L, Schimdt MD, Roberts DE, Hosmer D, Markenson G, Freedson PS. Development and validatium of a pregnancy physical activity questionnary. Med Sci Sports Exerc. 2004;36:1750-60.

20. Melo ASO, Assunção PL, Gondim SSR, Carvalho DF, Amorim MMR, Benício MHD'A, et al. Estado nutricional materno, ganho de peso gestacional e peso ao nascer. Rev Bras Epidemiol. 2007;10:249-57.

21. Assunção PL, Melo ASO, Gondim SSR, Benicio MHD, Amorin MMR, Cardoso MAA. Ganho ponderal e desfechos gestacionais em mulheres atendidas pelo Programa de Saúde da Família em Campina Grande, PB (Brasil). Rev Bras Epidemiol. 2007; 7:352-60

22. Nucci LB, Duncan BB, Mengue SS. Avaliação de ganho ponderal intragestacional em serviços de assistência pré-natal no Brasil. Cad Saude Pública 2001;17:1367-74.

23. Aquino KKNC. Determinantes do ganho ponderal excessivo em gestantes atendidas nos serviços públicos de pré-natal do distrito federal [dissertação]. Brasília (DF): Universidade de Brasília - UNB; 2004. 
24. Andreto LM, Souza AI, Figueiroa JN, Cabral-Filho JE. Fatores associados ao ganho ponderal excessivo em gestantes atendidas em um serviço público de pré-natal na cidade de recife, Pernambuco, Brasil. Cad Saude Pública. 2006;22:2401-09.

25. Prevedel TTS, Calderon IMP, De Conti MH, Consonni EB, Rudge MVC. Repercussões maternas e perinatais da hidroterapia na gravidez. Rev Bras Ginecol Obstet. 2003;25:53-9.

26. Monteiro CA, Benício, MHD’A, Ortiz LP. Tendência secular do peso ao nascer na cidade de São Paulo (1976-1998). Rev Saude Pública 2000;34:26-40.

27. Kac G, Velásquez-Meléndez G. Ganho de peso gestacional e macrossomia em uma coorte de mães e filhos. J Pediatr. 2005;81:47-53

28. Tomić V, Bosnjak K, Petrov B, Dikić M, Knezević D. Macrosomic births at mostar clinical hospital: a 2-year review. Bosn J Basic Med Sci. 2007;7:271-4.

29. Bergman RL, Richter R, Bergman KE, Plagemann A, Brauer M, Dudenhausen JW. Secular trends in neonatal macrosomia in Berlin. Influences of potential determinants. Paediatr Perinat Epidemiol. 2003;17:244-9.
30. Orr ST, James SA, Garry J, Prince CB, Newton ER Exercise and pregnancy outcome among urban, low-income, black women. Ethn Dis. 2006;16:933-7.

31. Hatch MC, ShuXO, Mclean DE. Maternal exercise during pregnancy, physical fitness, and fetal growth. Am J Epidemiol. 1993;137:1105-14.

32. Lindseth G, Vari P. Measuring physical activity during pregnancy. West J Nurs Res. 2005;27:722-34.
Artigo recebido: 23/06/08 Aceito para publicação: 06/11/08 